\title{
Discrete Mode Lasers for Applications in Access Networks
}

\author{
A. Kaszubowska-Anandarajah, L. Barry, P. Anandarajah, C. Guignard, B. Kelly *, J. O'Gorman* \\ Research Institute for Networks and Communications Engineering (RINCE), Radio \& Optical Communication \\ Laboratory, Dublin City University (DCU), Ireland, e-mail: aleksandra kaszubowska@dcu.ie \\ * Eblana Photonics Ltd, Unit 32, Trinity Enterprise Centre, Dublin 2, Ireland.
}

\begin{abstract}
Fast development of the modern telecommunication networks such as fiber-to-the-home or radio-over-fiber systems require an inexpensive y et reliable optical transmitter for electro-optic conversion. Such devices should be able to generate stable, single moded optical signals suitable for athermal operation. Discrete Mode Lasers (DMLs) are able to fulfill all the above-mentioned requirements with the added benefit of low sensitivity to optical feedback. DMLs are essentially Fabry-Perot lasers in which the refractive index is modified by introducing perturbations along very small sections of the laser cavity. These modifications result in a single mode laser output with a very narrow linewidth (order of $400 \mathrm{kHz}$ ). In this paper, we demonstrate how a DML can outperform the commonly used/commercially available DFB lasers in terms of linewidth, sensitivity to optical feedback and transmission performance in the presence of feedback.
\end{abstract}

Keywords: Radio-over-Fiber systems, discrete mode lasers, direct modulation, optical feedback, Fabry-Perot lasers, DFB lasers

\section{INTRODUCTION}

Wide deployment of broadband access networks that employ Radio-over-Fiber (RoF) technology depends strongly on the cost of system components [1]. The availability of an inexpensive, single wavelength laser diode would help to make the access networks more affordable to the service providers and as a result to end users. The reduction in price could be achieved by simplifying the fabrication process and the packaging requirements of the laser transmitter.

Amongst many types of single mode lasers, the most commonly used are the Distributed FeedBack (DFB) laser and the Vertical Cavity Surface Emitting Laser (VCSEL). However, the uniform fabrication of such lasers with predictable spectra remains a challenge [2]. In addition to this stringent fabrication conditions, most of the semiconductor lasers require the introduction of an optical isolator between the laser and the coupling fiber in order to avoid performance degradation due to optical feedback [3]. All the above-mentioned problems lead to an increase in the cost of both DFB and VCSEL lasers, which make these devices non-ideal for price sensitive access and enterprise applications [2].

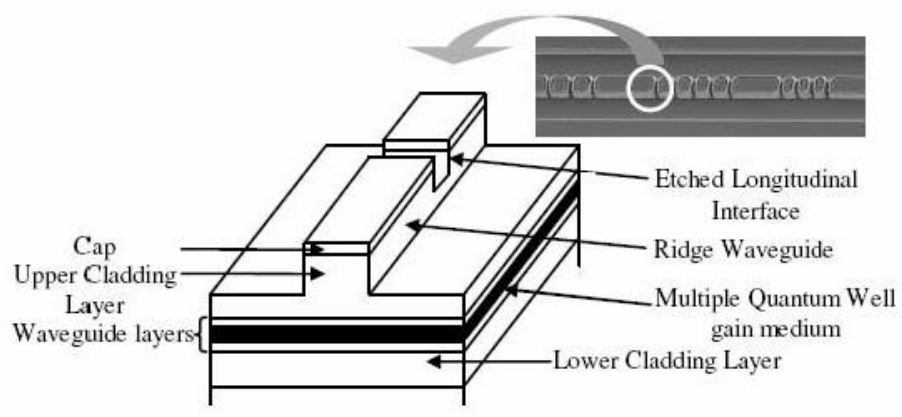

Figure 1. Fabry-Perot laser incorporating a single index perturbation in the form.

An alternative to DFB lasers and VCSELs are Discrete Mode (DM) lasers. The concept of these lasers was built on the work of DeChiaro who demonstrated that the emission spectrum of a laser diode can be altered by introducing one or more artificial index perturbations in the laser cavity [4]. DM lasers are then obtained from ridge waveguide Fabry Perot (FP) lasers, in which the effective refractive index of the lasing mode has been perturbed (see Fig. 1). The perturbation of this index can be achieved by etching features into the ridge waveguide such that each feature has a small overlap with the transverse field profile of the unperturbed mode. Most of the light in the laser cavity is therefore unaffected by these perturbations whereas a proportion of the propagating light is reflected at the boundaries between the perturbed and the unperturbed sections (Fig. 2). It has been demonstrated that suitable positioning of these interfaces allows the mirror loss spectrum of a FP laser to be manipulated in order to achieve single longitudinal mode emission (by reducing the threshold gain of an individual cavity mode below that of the other cavity modes) [5].

This work is $t$ carried out under Science Foundation Ireland Industrial Supplement Grant 
DM laser diodes are capable of single mode operation over wide temperature ranges and exhibit Side Mode Suppression Ratios (SMSR) in excess of $30 \mathrm{~dB}$ [5]. Another important advantage of the DM lasers is their immunity to optical feedback [6]. This feature removes the requirement of placing an isolator at the output of the device. This attribute, together with the simpler fabrication process, results in the reduced cost of this component.

In this paper, we examine the performance of a DM laser employed as a transmitter in a RoF system, in particular its immunity to external feedback, and compare it with the performance of a commercially available DFB laser diode.

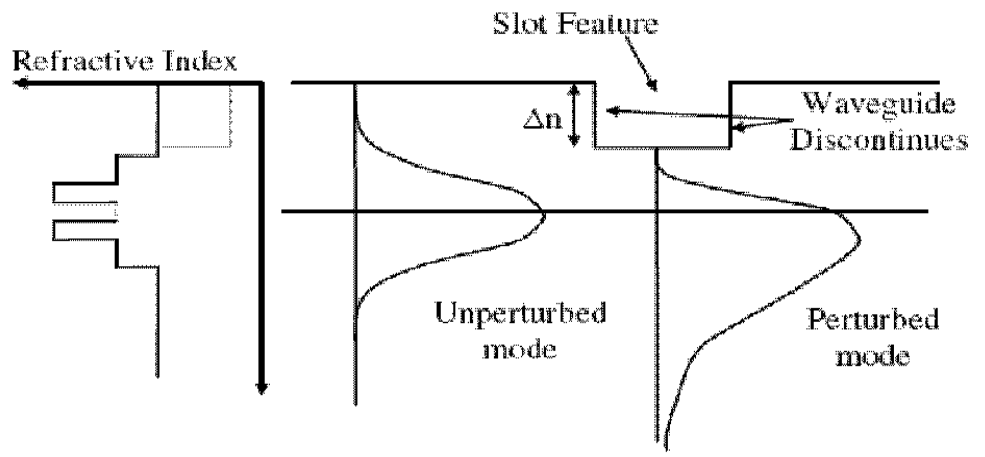

Figure 2. Schematic illustrating the effect of a slot feature on the transverse.

\section{EXPERIMENTAL SET-UP}

The experimental set-up used to examine the impact of feedback on the laser transmitted in a RoF system is shown in Fig. 3. The set-up consists of a laser diode (DM and subsequently DFB laser), which is directly modulated with $155 \mathrm{Mb} / \mathrm{s}$ data upconverted to $2.4 \mathrm{GHz}$. A polarization controller is placed at the output of the diode in order to maximize the impact of the feedback on the laser performance. The light from the laser is then split using a 90:10 coupler. The source of the optical feedback is a reflector connected to the $90 \%$ arm of the coupler. The level of the reflected light is varied using a variable attenuator. The effective feedback level that returns to the laser chip depends on the coupling efficiency of the device to the fiber. The DM laser has a better coupling efficiency than the DFB laser, resulting in a coupling loss of $2.6 \mathrm{~dB}$ (3.5 $\mathrm{dB}$ for the DFB laser used).

The signal from the $10 \%$ arm of the coupler passes through an isolator and is subsequently launched into $10 \mathrm{~km}$ of single mode fiber. The signal is then amplified using an EDFA and passed through a $2 \mathrm{~nm}$ Optical Band Pass Filter (OBPF). The optical power falling on the receiver is varied using a variable optical attenuator. The receiver consists of an EDFA (giving a constant output power of $6 \mathrm{dBm}$ ), a $1 \mathrm{~nm} \mathrm{OBPF,} \mathrm{90:10} \mathrm{coupler} \mathrm{and}$ a photodiode. The detected RF signal is electrically amplified, downconverted to baseband, filtered and displayed using an oscilloscope. In order to verify the performance of the two different lasers subjected to feedback, BER measurements were carried out as a function of the feedback level.

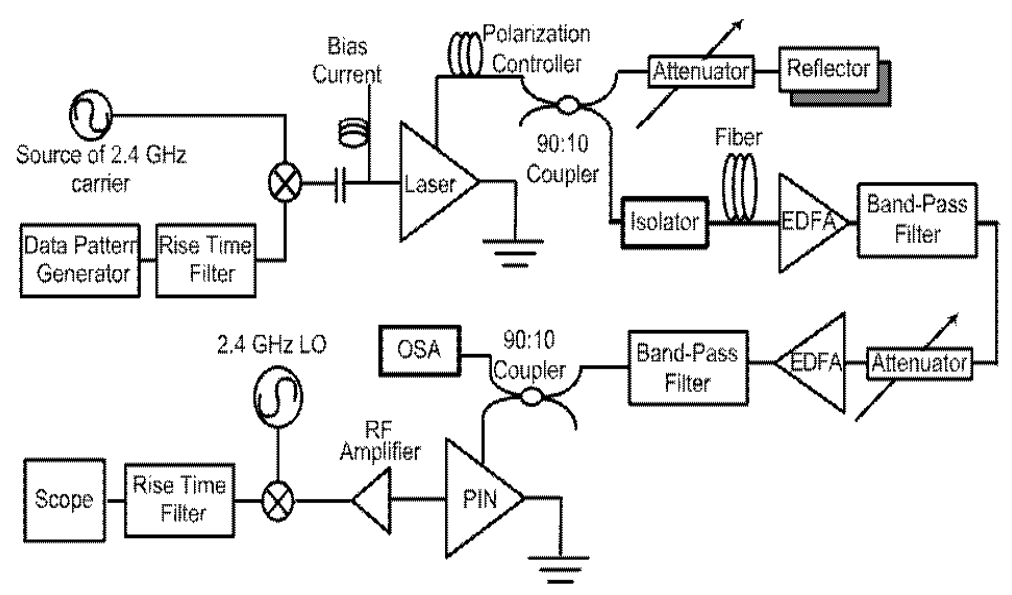

Figure 3. Experimental set-up.

The PI curves of both lasers are shown in Fig. 4. It can be seen that both lasers have near identical slopes of their PI curve $(0.096 \mathrm{~W} / \mathrm{A})$. The threshold current of the DM and the DFB laser was 14 and $10 \mathrm{~mA}$ respectively and the bias currents were chosen to give equal output power $(2 \mathrm{~mW})$ for both lasers ( $36 \mathrm{~mA}$ for the DM and $30 \mathrm{~mA}$ for the DFB laser). The experiments were carried out for two Modulation Indices (MIs) of 28 and $59 \%$ and the received power was set to obtain a BER of $10^{-10}$ for $59 \%$ MI without any feedback. For $28 \%$ MI 
the signal to noise ratio is reduced, therefore the best BER that can be achieved at the same received power is $10^{-8}$

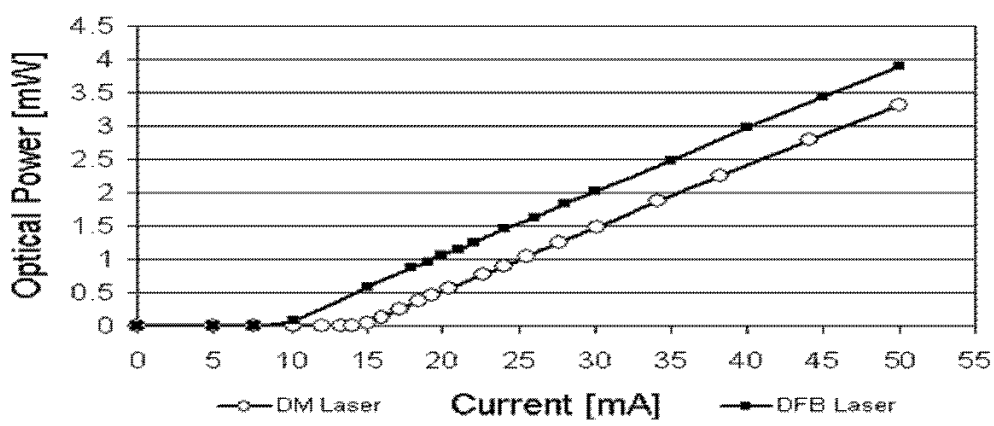

Figure 4. PI curves of the DM and DFB laser.

\section{EXPERIMENTAL RESULTS}

The BER vs. feedback level measurements for DM and DFB lasers for 59 and $28 \%$ modulation index are shown in Fig. 5 and 6 respectively. In the first case low values of feedback have a negligible impact on the performance of both lasers. As the feedback level exceeds $-35 \mathrm{dBm}$ there is a slight deterioration in the BER associated with the DM laser, while the BER linked to the DFB laser starts to degrade drastically. Feedback level of $-20 \mathrm{dBm}$ is required to observe similar signal degradation in the case of the DM laser. At a reference BER of $10^{-8}$, the DM laser is capable of handling $10 \mathrm{~dB}$ higher levels of optical feedback in comparison to the DFB laser.

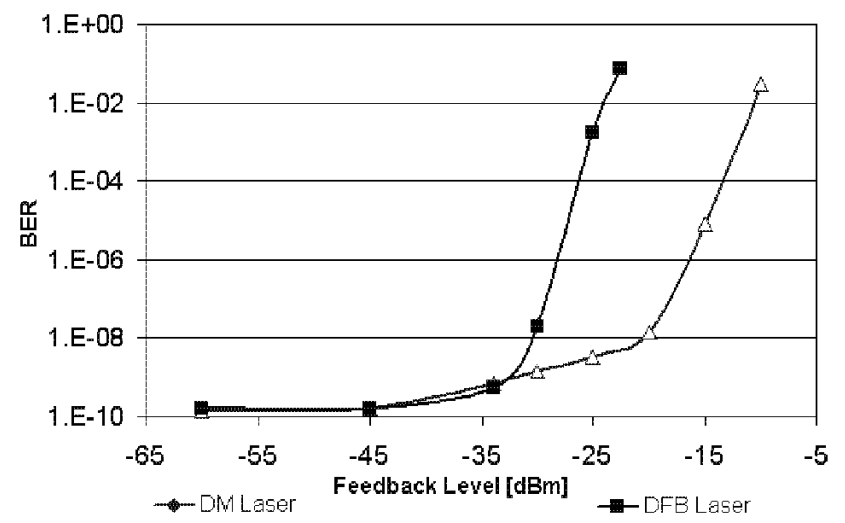

Figure 5. BER vs. feedback level for $D M$ and DFB laser for $59 \% M I$.

For a smaller MI, the difference in performance between both lasers is smaller. Nevertheless, the DM lasers exhibits a better immunity to feedback than the DFB laser $\left(5 \mathrm{~dB}\right.$ for BER of $\left.10^{-8}\right)$.

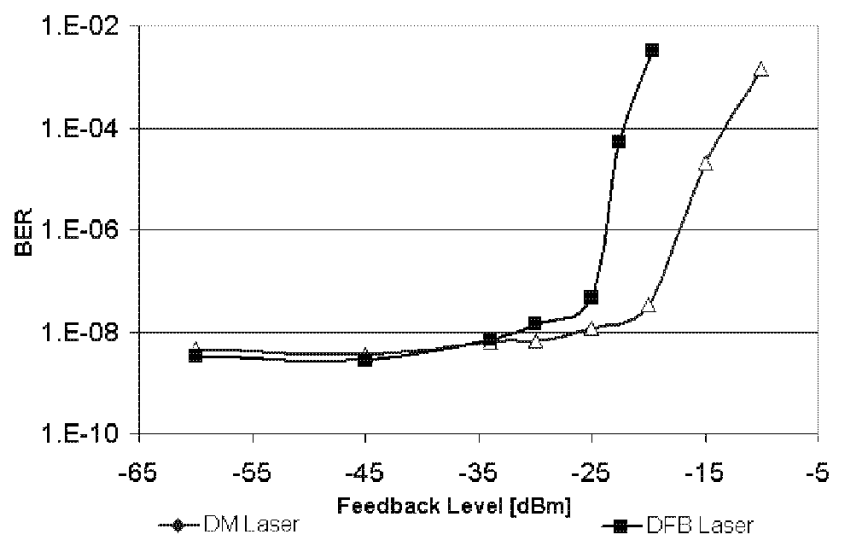

Figure 6. BER vs. feedback level for DM and DFB laser for $28 \% \mathrm{MI}$.

Figure $7 a$ and $7 b$ present the optical spectra of the DM and DFB lasers respectively for different levels of optical feedback. The split in the spectrum of the DFB laser indicates that mode hopping occurs. On the other hand for the DM laser the mode hopping is suppressed.

The fact that DM lasers are less sensitive to optical feedback than DFB lasers can be explained by the structure of the laser itself. In DFB lasers, two grating modes are in competition with the cavity (FP) modes, whereas DM 
lasers are truly single mode lasers. In the case of DFB lasers, the lasing of only one of the grating modes (yielding a stable emission spectrum) could be achieved by having one facet with high reflective coating and the other with antireflection coating. However, the AR-coated emitting facet allows more optical feedback to enter the laser chip causing large mode partition noise. Thus the structure of DFB lasers is susceptible to mode partition noise when light is reflected back into the laser.
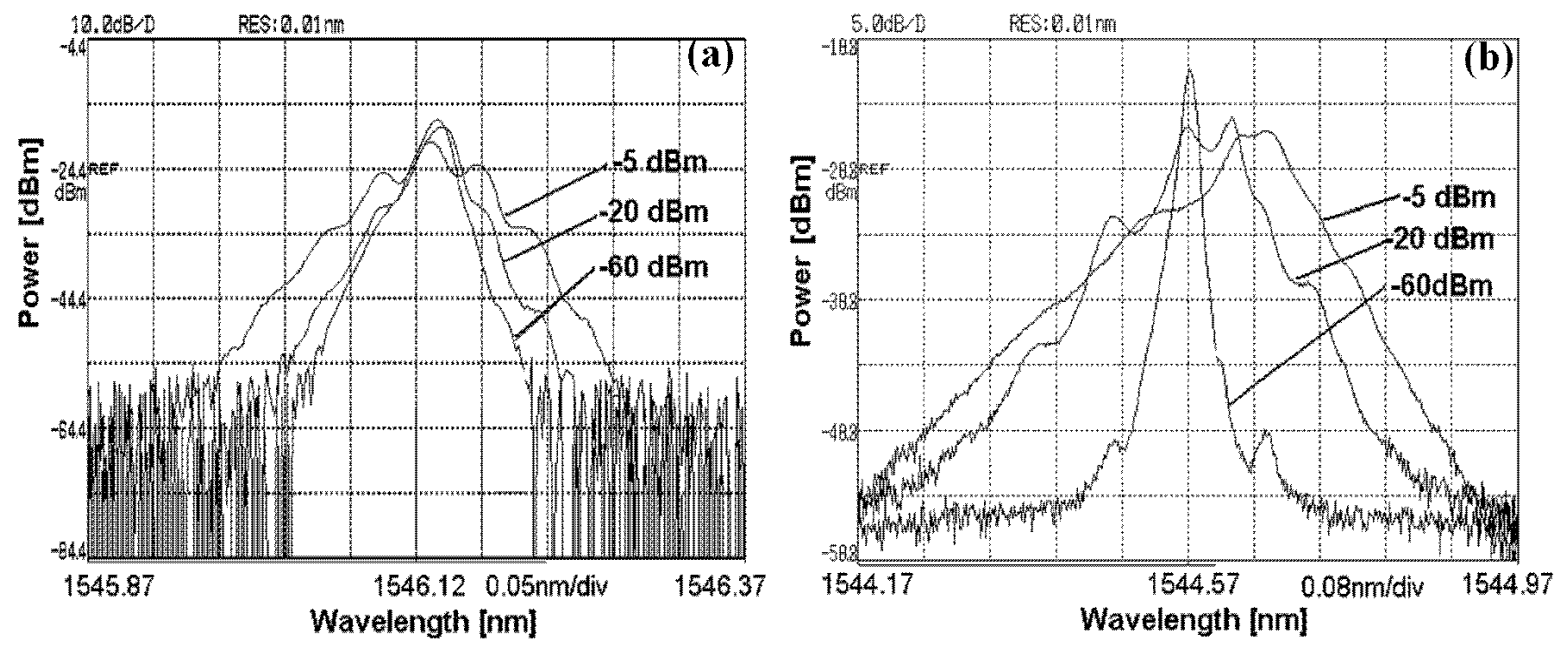

Figure 7. Optical spectra for different feedback levels for (a) DM laser, (b) DFB laser.

\section{CONCLUSIONS}

The availability of reliable and inexpensive components is of paramount importance for the development of modern access networks such as FTTH or RoF. While the manufacturing of VCSEL and DFB lasers remains a relatively complicated and costly process, it is important to look for other solutions. DM lasers might prove to be just that, they provide high SMSR, low linewidth and capability to operate over large temperature ranges, while their manufacturing process is limited to a standard optical lithography.

This paper explored an additional advantage of the DM lasers, which is low sensitivity to optical feedback. The comparative study between the DM and DFB lasers shows that, in RoF distribution system, for 59\% MI the DM laser can handle $10 \mathrm{~dB}$ more optical feedback than DFB at a reference BER of $10^{-8}$. This is a direct effect of the laser structure that ensures a truly single mode operation of the device, therefore suppressing mode hopping.

\section{REFERENCES}

[1] R. P. Braun: Fiber Rdio Systems, Applications and Devices, ECOC'98 Tutorial.

[2] C.J. Chang-Hasnain: Tunable VCSEL, IEEE.J. Quantum Electronics, vol. 6, pp. 978-97, 2000.

[3] R. Tkach: Regimes of Feedback Effects in 1.5-pm Distributed Feedback Lasers, IEEE J. Lightwave Technol., vol. 4, no. 11, pp. 1655-1661, 1986.

[4] L. DeChiaro: Damage Induced Spectral Perturbations in Multilongitudinal-Mode Semiconductor Lasers, IEEE J. Lightwave Technol., vol. 8, no. 11, pp. 1659-1669, 1990.

[5] J. Patchell D. Jones, B. Kelly, J. O'Gorman: Specifying the wavelength and temperature tuning range of a Fabry-Perot laser containing refractive index perturbations, Proc. SPIE 5825, 1, 2005.

[6] C. Guignard G. Tipaka, O. Vaudel, B. Kelly, J. Patchell, L. Barry, P. Besnard and J. O'Gorman: Low Sensitivity to Optical Feedback and Optical Injection of Discrete Mode Lasers, Proc. LEOS (2006), pp. 663-664 